Additional Perspectives articles for Influenza: The Cutting Edge book collection are available at http://perspectivesinmedicine.cshlp.org/cgi/collection/influenza_the_cutting_edge.

\title{
Influenza Polymerase Inhibitors: Mechanisms of Action and Resistance
}

\author{
Emi Takashita \\ Influenza Virus Research Center, National Institute of Infectious Diseases, Musashimurayama, \\ Tokyo 208-0011, Japan \\ Correspondence: emitaka@nih.go.jp
}

\begin{abstract}
The influenza virus RNA-dependent RNA polymerase is highly conserved among influenza A, B, C, and D viruses. It comprises three subunits: polymerase basic protein 1 (PB1), polymerase basic protein 2 (PB2), and polymerase acidic protein (PA) in influenza $A$ and $B$ viruses or polymerase 3 protein $(\mathrm{P} 3)$ in influenza $C$ and $D$ viruses. Because this polymerase is essential for influenza virus replication, it has been considered as a target for antiviral agents. Recently, several polymerase inhibitors that target each subunit have been developed. This review discusses the mechanism of action, antiviral activity, and emergence of resistance to three inhibitors approved for the treatment of influenza or in late-phase clinical trials: the PB1 inhibitor favipiravir, the PB2 inhibitor pimodivir, and the PA inhibitor baloxavir marboxil.
\end{abstract}

$\mathrm{T}^{\mathrm{h}}$ he influenza virus RNA-dependent RNA polymerase is highly conserved among influenza A, B, C, and D viruses (Peng et al. 2019). It consists of three subunits: polymerase basic protein 1 (PB1), polymerase basic protein 2 (PB2), and polymerase acidic protein (PA) in influenza $A$ and $B$ viruses or polymerase 3 protein (P3) in influenza $C$ and D viruses (Reich et al. 2014; Hengrung et al. 2015; Fan et al. 2019; Peng et al. 2019).

The RNA-dependent RNA polymerase is essential for the viral replication cycle, including transcription and viral protein synthesis, as well as viral genome replication (Stevaert and Naesens 2016). Primary transcription of the viral genome RNA (vRNA) to mRNA starts with the cap-snatching reaction, in which the $5^{\prime}$-terminal 7-methylguanosine $\left(\mathrm{m}^{7} \mathrm{G}\right)$ cap structure of the host mRNA is bound by the cap-binding domain of PB2 and cleaved by the cap-dependent endonuclease of PA at 10-13 nucleotides from the cap, to yield primers for viral mRNA synthesis. Replication of vRNA then proceeds to yield the full-length complementary RNA (cRNA). During both transcription and replication, RNA elongation is carried out by PB1.

The polymerase active sites for RNA binding, cleavage, and elongation are conserved among the various types of influenza viruses, which is why the RNA-dependent RNA polymerase has been considered as a target for antiviral agents. Recently, several polymerase inhibitors that target each subunit have been developed. This review provides an overview of three such inhibitors approved for the treatment of influenza or in late-phase clinical trials: the PB1 inhibitor favipiravir, the PB2 inhibitor pimodivir, and the PA inhibitor baloxavir marboxil (Table 1).

Editors: Gabriele Neumann and Yoshihiro Kawaoka

Additional Perspectives on Influenza: The Cutting Edge available at www.perspectivesinmedicine.org

Copyright (C) 2021 Cold Spring Harbor Laboratory Press; all rights reserved; doi: 10.1101/cshperspect.a038687

Cite this article as Cold Spring Harb Perspect Med 2021;11:a038687 
E. Takashita

Table 1. Overview of influenza polymerase inhibitors

\begin{tabular}{|c|c|c|c|}
\hline & Favipiravir & Pimodivir & Baloxavir marboxil \\
\hline Alternative name & $\mathrm{T}-705$ & $\begin{array}{l}\text { JNJ-63623872, VX- } \\
\quad 787\end{array}$ & S-033188 \\
\hline Molecular formula & $\mathrm{C}_{5} \mathrm{H}_{4} \mathrm{FN}_{3} \mathrm{O}_{2}$ & $\mathrm{C}_{20} \mathrm{H}_{19} \mathrm{~F}_{2} \mathrm{~N}_{5} \mathrm{O}_{2}$ & $\mathrm{C}_{27} \mathrm{H}_{23} \mathrm{~F}_{2} \mathrm{~N}_{3} \mathrm{O}_{7} \mathrm{~S}$ \\
\hline Molecular weight & 157.1 & 399.4 & 571.6 \\
\hline Active form & Favipiravir-RTP & - & Baloxavir acid \\
\hline Target protein & PB1 & PB2 & PA \\
\hline Target influenza viruses & Types A, B, C, D & Type A & Types A, B, C, D \\
\hline Current status & $\begin{array}{l}\text { Approved in Japan for } \\
\text { influenza } \\
\text { pandemic } \\
\text { preparedness }\end{array}$ & $\begin{array}{l}\text { In Phase III clinical } \\
\text { trials }\end{array}$ & $\begin{array}{l}\text { Approved in several } \\
\text { countries (Japan, } \\
\text { USA, etc.) }\end{array}$ \\
\hline Route of administration & BID oral dose for $5 \mathrm{~d}$ & BID oral dose for $5 \mathrm{~d}$ & Single oral dose \\
\hline Inhibition of M2 inhibitor-resistant viruses & Yes & Yes & Yes \\
\hline Inhibition of NA inhibitor-resistant viruses & Yes & Yes & Yes \\
\hline $\begin{array}{l}\text { Emergence of variants with reduced } \\
\text { susceptibility in vitro }\end{array}$ & Yes & Yes & Yes \\
\hline $\begin{array}{l}\text { Emergence of variants with reduced } \\
\text { susceptibility in clinical trials }\end{array}$ & No & Yes & Yes \\
\hline
\end{tabular}

(Favipiravir-RTP) Favipiravir-ribofuranosyl-5'-triphosphate, (PB1) polymerase basic protein 1, (PB2) polymerase basic protein 2, (PA) polymerase acidic protein, (BID) twice daily, (M2) matrix protein 2, (NA) neuraminidase.

\section{FAVIPIRAVIR}

Favipiravir (T-705) was developed in Japan and approved for influenza pandemic preparedness in Japan on March 24, 2014 (http://www.pmda .go.jp/files/000210319.pdf). Because favipiravir increases the risk for teratogenicity and embryotoxicity, conditional marketing approval with strict regulations for its production and clinical use was granted (Furuta et al. 2017). Accordingly, favipiravir is available only for patients with novel or reemerging pandemic influenza virus infection in whom other influenza antivirals are ineffective or not sufficiently effective. In other countries, favipiravir is under investigation. Two multinational phase 3 clinical trials of favipiravir in adults with uncomplicated influenza were completed in 2015 (NCT02008344; NCT020 26349). A phase 2 a clinical trial of favipiravir in hospitalized patients aged $18 \mathrm{yr}$ and older with severe influenza was completed in China in March 2019 (NCT03394209).

\section{Mechanism of Action}

Favipiravir, a pyrazine derivative, was discovered through the screening of a chemical library for compounds with in vitro anti-influenza virus activity (Furuta et al. 2002). Favipiravir undergoes phosphoribosylation and further phosphorylation in infected cells to achieve its active form, favipiravir-ribofuranosyl- $5^{\prime}$-triphosphate (favipiravir-RTP) (Furuta et al. 2005).

Favipiravir acts as a chain terminator or a mutagen (Vanderlinden et al. 2016; Goldhill et al. 2019). It is recognized as a purine analog by the RNA-dependent RNA polymerase and efficiently incorporated into a nascent RNA strand as a guanosine and an adenosine analog (Jin et al. 2013; Sangawa et al. 2013). This prevents the incorporation of GTP and ATP in a competitive manner and inhibits RNA elongation, which is carried out by PB1. Furthermore, favipiravir causes lethal mutagenesis by increasing the G-to-A and C-to-U mutation frequency and generating nonviable progeny virus (Baranovich et al. 2013).

\section{Antiviral Activity}

Favipiravir has activity against the RNA-dependent RNA polymerase of influenza and other RNA viruses but not human DNA-dependent 
RNA and DNA polymerases (Furuta et al 2013). Favipiravir inhibits the replication of influenza $A, B, C$, and $D$ viruses, including matrix protein 2 (M2) inhibitor-resistant viruses and neuraminidase (NA) inhibitor-resistant viruses (Furuta et al. 2002; Mishin et al. 2019).

Synergistic effects of favipiravir and an NA inhibitor (oseltamivir, peramivir, zanamivir, or laninamivir) have been demonstrated in vitro (Tarbet et al. 2014) and in mice (Smee et al. 2010, 2013; Tarbet et al. 2012; Park et al. 2014; Marathe et al. 2016; Baz et al. 2018; Kiso et al. 2018). A phase 2 a clinical trial of favipiravir suggested that combination therapy of favipiravir and oseltamivir may accelerate clinical recovery in hospitalized patients aged $18 \mathrm{yr}$ and older with severe influenza (Wang et al. 2019).

\section{Resistance}

Ten in vitro passages of A/England/195/2009 (H1N1)pdm09 virus in the presence of favipiravir led to the acquisition of a K229R substitution in PB1 that conferred a 31-fold reduction in susceptibility to favipiravir (Goldhill et al. 2018). The PB1 K229R substitution prevented the incorporation of favipiravir into nascent viral RNA and decreased the mutation frequency in the presence of favipiravir (Goldhill et al. 2018). Although the PB1 K229R substitution significantly reduced the viral polymerase activity and replication fitness, a P653L substitution in PA compensated for the fitness costs. The PB1 K229R substitution is conserved among different influenza subtypes and confers reduced favipiravir susceptibility to the RNA-dependent RNA polymerase of A/Victoria/3/1975(H3N2) and A/Anhui/1/2013(H7N9) viruses (Goldhill et al. 2018).

In a mouse model infected with the highly pathogenic A/Turkey/15/2006(H5N1) virus, emergence of resistance was not observed in both the favipiravir monotherapy and favipiravir plus oseltamivir combination therapy groups (Marathe et al. 2016). In immunodeficient mice infected with A/Quebec/144147/2009(H1N1) pdm09 virus (Baz et al. 2018) and immunocompromised nude mice infected with A/California/ 04/2009(H1N1)pdm09 virus (Kiso et al. 2018), combination therapy with favipiravir and NA inhibitor, oseltamivir, or laninamivir did not induce variants with reduced susceptibility to favipiravir, although variants with reduced susceptibility to the NA inhibitors did emerge.

In phase 3 clinical trials of favipiravir, some amino acid substitutions in PB1, PB2, and/or PA emerged after favipiravir treatment (Takashita et al. 2016). A L666F substitution in PA conferred reduced polymerase activity to $\mathrm{A}(\mathrm{H} 1 \mathrm{~N} 1)$ pdm09 virus, although no variants with reduced susceptibility to favipiravir emerged during the clinical trials.

\section{PIMODIVIR}

Pimodivir (JNJ-63623872, VX-787) received Fast Track designation by the Food and Drug Administration (FDA) in March 2017 because of its potential to address an unmet medical need in those who develop influenza A infection and are hospitalized or at high risk of related complications (Finberg et al. 2019). Two phase 3 clinical trials of pimodivir are ongoing in hospitalized patients and high-risk outpatients aged 13-85 yr with influenza A infection (NCT03376321; NCT03381196). In both clinical trials, the efficacy and safety of pimodivir in combination with the standard-of-care treatment, which may include influenza antivirals and/or supportive care only, are being evaluated.

\section{Mechanism of Action}

Pimodivir is a cyclohexyl carboxylic acid analog obtained by screening a compound library for compounds with in vitro activity against influenza virus (Clark et al. 2014). In the cap-snatching reaction, $\mathrm{PB} 2$ binds to the $\mathrm{m}^{7} \mathrm{G}$ cap structure of host mRNA (Stevaert and Naesens 2016). Pimodivir occupies the cap-binding domain of PB2 and inhibits RNA binding (Clark et al. 2014; Byrn et al. 2015; Stevaert and Naesens 2016). Pimodivir forms hydrogen bonds with E361 and K376 in the PB2 cap-binding domain. The azaindole ring of pimodivir is sandwiched between $\mathrm{H} 357$ and F404, whereas the pyrimidine ring interacts with F323. The carboxylic acid of pimodivir makes water-mediated inter- 
E. Takashita

actions with R355, H357, and Q406 (Clark et al. 2014; Byrn et al. 2015).

\section{Antiviral Activity}

Pimodivir has activity against influenza A viruses, including M2 inhibitor-resistant viruses and NA inhibitor-resistant viruses (Clark et al. 2014; Byrn et al. 2015), but showed negligible activity against influenza B viruses (Byrn et al. 2015). Several key amino acids in the PB2 cap-binding domain differ between influenza $A$ and $B$ viruses (Liu et al. 2015), and some residues in the cap-binding domain of influenza B virus could sterically hinder pimodivir (Severin et al. 2016). Furthermore, the cap-binding affinity and specificity of influenza $B$ virus differ from those of influenza A virus (Liu et al. 2015): influenza B virus has weaker cap-binding affinity than influenza A virus (Wakai et al. 2011); and influenza A virus has strict specificity for the $m^{7} \mathrm{G}$ cap structure, whereas influenza $B$ virus recognizes the unmethylated cap structure (Wakai et al. 2011).

Synergistic effects have been demonstrated in vitro for pimodivir and favipiravir, oseltamivir, or zanamivir (Byrn et al. 2015). In the phase 2b TOPAZ clinical trial of pimodivir (NCT02 342249), combination therapy of pimodivir plus oseltamivir resulted in a significantly lower viral load compared with pimodivir monotherapy in adults with acute uncomplicated influenza A (Finberg et al. 2019). The phase 2b OPAL clinical trial of pimodivir (NCT02532283), which compared the combination therapy of pimodivir plus oseltamivir to oseltamivir monotherapy, showed shorter durations of virus detection and illness for the combination therapy compared with oseltamivir monotherapy in hospitalized patients aged 18-85 yr enrolled within $72 \mathrm{~h}$ of symptom onset (Hayden and Shindo 2019; Mifsud et al. 2019).

\section{Resistance}

In vitro passages of A/Puerto Rico/8/34(H1N1) virus in the presence of pimodivir identified six amino acid substitutions in PB2 (i.e., Q306H, S324I, S324N, S324R, F404Y, and N510T) that were associated with reduced susceptibility to pimodivir (Byrn et al. 2015). These substitutions conferred 63- to 257-fold reduced susceptibility to pimodivir and are rare in naturally occurring human influenza viruses $(0 \%-0.03 \%)$, suggesting that they are treatment-emergent substitutions.

In a mouse model of A/Puerto Rico/8/34 (H1N1) virus infection, emergence of resistance was not observed (Byrn et al. 2015).

In a phase $2 \mathrm{a}$ clinical trial of pimodivir, potential pimodivir-resistant variants with amino acid substitutions in PB2 (i.e., S324C, K376R, M431L/R/V, and M431I) were identified between days 3 and 7 of treatment in 9.7\% (7/72) of pimodivir-treated volunteers infected with $\mathrm{A} /$ Wisconsin/67/2005(H3N2) virus (Trevejo et al. 2018). These volunteers had both high viral titers and high mean steady state minimum pimodivir concentrations in the plasma. The PB2 M431I substitution was detected in four of seven volunteers and conferred 57-fold reduced susceptibility to pimodivir, which was associated with a 13-fold reduction in replication capacity compared with the wild-type virus. Emergence of these substitutions was not coincident with viral rebound and all subjects subsequently cleared the virus after treatment for $5 \mathrm{~d}$ once daily.

In the phase $2 \mathrm{~b}$ TOPAZ clinical trial of pimodivir, $\mathrm{A}(\mathrm{H} 1 \mathrm{~N} 1) \mathrm{pdm} 09$ or $\mathrm{A}(\mathrm{H} 3 \mathrm{~N} 2)$ viruses with $\mathrm{PB} 2$ substitutions or reduced susceptibility to pimodivir were detected in $6.9 \%$ (4/58), $10.5 \%$ $(6 / 57)$, and $1.8 \%(1 / 57)$ of patients treated with pimodivir $300 \mathrm{mg}$, pimodivir $600 \mathrm{mg}$, and a combination of pimodivir $600 \mathrm{mg}$ and oseltamivir, respectively (Finberg et al. 2019). The PB2 substitutions detected in these patients (i.e., S324K/N/R, F325L, S337P, K376N/R, T378S, and $\mathrm{N} 510 \mathrm{~K}$ ) were associated with 9.4- to $>372$ fold reduced susceptibility to pimodivir.

\section{BALOXAVIR MARBOXIL}

Baloxavir marboxil (S-033188) was developed in Japan and approved there on 23 February 2018 for the treatment of influenza $A$ and $B$ virus infection in patients aged $12 \mathrm{yr}$ and older and children younger than $12 \mathrm{yr}$ weighing $\geq 10 \mathrm{~kg}$ (http://www.pmda.go.jp/files/000225380.pdf). 
Table 2. Influenza antiviral supply to medical institutes in Japan

\begin{tabular}{|c|c|c|c|c|c|}
\hline \multirow[b]{3}{*}{ Influenza season } & \multicolumn{5}{|c|}{ Doses $(\times 10,000)$} \\
\hline & \multicolumn{4}{|c|}{ NA inhibitors } & \multirow{2}{*}{$\frac{\text { Polymerase inhibitor }}{\text { Baloxavir }}$} \\
\hline & Zanamivir & Oseltamivir & Peramivir & Laninamivir & \\
\hline 2009-2010 & 515 & 859 & 0.5 & - & - \\
\hline 2010-2011 & 54 & 240 & 13 & 166 & - \\
\hline 2011-2012 & 127 & 356 & 26 & 270 & - \\
\hline 2012-2013 & 110 & 358 & 35 & 280 & - \\
\hline 2013-2014 & 151 & 336 & 37 & 330 & - \\
\hline 2014-2015 & 132 & 422 & 37 & 400 & - \\
\hline 2015-2016 & 40 & 375 & 40 & 368 & - \\
\hline 2016-2017 & 151 & 453 & 50 & 485 & - \\
\hline 2017-2018 & 158 & 570 & 60 & 690 & 40 \\
\hline 2018-2019 & 59 & 464 & 32 & 289 & 528 \\
\hline
\end{tabular}

Data adapted from notification on stable supply of influenza antivirals from the Ministry of Health, Labour, and Welfare, Japan.

(NA) Neuraminidase, (-) unapproved.

The FDA granted a Priority Review to baloxavir, under which the FDA takes expedited action on an application to determine whether the drug, if approved, would significantly improve the safety or effectiveness of treating, diagnosing, or preventing a serious condition. Indeed, baloxavir was approved in the United States on 24 October 2018 (https://www.accessdata.fda.gov/drugsatfda docs/nda/2018/210854Orig1s000TOC.cfm) and in several other countries in 2019 for patients aged $12 \mathrm{yr}$ and older weighing at least $40 \mathrm{~kg}$. Because it is not clear whether the selected doses of $40 \mathrm{mg}$ or $80 \mathrm{mg}$ for subjects weighing $\geq 80 \mathrm{~kg}$ are adequate to control influenza $B$ virus infections, the FDA has recommended that higher doses of baloxavir be evaluated (https://www .accessdata.fda.gov/drugsatfda_docs/nda/2018/ 210854Orig1s000MicroR.pdf). The phase 3 MINISTONE-2 clinical trial of baloxavir in patients 1 to $<12 \mathrm{yr}$ of age was completed in the United States and other countries in April 2019 (NCT03629184). In this trial, higher doses of baloxavir ( $2 \mathrm{mg} / \mathrm{kg}$ for subjects weighing $<20 \mathrm{~kg}$ and $40 \mathrm{mg}$ for $\geq 20 \mathrm{~kg}$ ), which were 2 - to 4-fold higher than those approved in Japan (10 mg for subjects weighing 10 to $<20 \mathrm{~kg}, 20 \mathrm{mg}$ for 20 to $<40 \mathrm{~kg}$, and $40 \mathrm{mg}$ for $\geq 40 \mathrm{~kg}$ ), were administered.

In the 2018-2019 influenza season, baloxavir was supplied to medical institutions that to- gether served about 5.3 million people in Japan (i.e., one-twenty-fourth of the Japanese population) and became the most used influenza antiviral in Japan (Table 2).

\section{Mechanism of Action}

Baloxavir acid (S-033447), an active form of baloxavir marboxil, was generated by rational molecular design based on the two-metal pharmacophore concept for dolutegravir, an inhibitor of human immunodeficiency virus (HIV) integrase, because influenza PA and HIV integrase both use two divalent metal ions as cofactors for their endonuclease activity (Omoto et al. 2018). To enhance oral absorption of baloxavir acid, a phenolic hydroxyl group was added, thereby creating baloxavir marboxil (Omoto et al. 2018).

Baloxavir marboxil is rapidly hydrolyzed by esterases in the intestine, liver, and blood into baloxavir acid (Koshimichi et al. 2019). Baloxavir acid binds to the PA endonuclease domain and inhibits RNA cleavage by the PA capdependent endonuclease (Omoto et al. 2018). Baloxavir acid forms van der Waals interactions with A20, Y24, K34, A37, and I38 in influenza A virus or with T20, F24, M34, N37, and I38 in influenza $\mathrm{B}$ virus in the PA endonuclease domain (Omoto et al. 2018). 
E. Takashita

\section{Antiviral Activity}

Baloxavir has activity against influenza A, B, C, and $\mathrm{D}$ viruses, including NA inhibitor-resistant viruses (Noshi et al. 2018; Mishin et al. 2019). Influenza B viruses are less susceptible to baloxavir than are influenza A viruses (Takashita et al. 2018; Gubareva et al. 2019; Koszalka et al. 2019); the susceptibility pattern is influenza $A>B>C>D$ (Mishin et al. 2019).

Synergistic effects of baloxavir and the NA inhibitor oseltamivir have been reported in vitro and in mice (Fukao et al. 2019). A phase 3 clinical trial of baloxavir to evaluate its efficacy and safety in combination with a standard-of-care NA inhibitor (oseltamivir, peramivir, or zanamivir) in hospitalized patients aged $12 \mathrm{yr}$ and older with severe influenza is currently recruiting patients (NCT03684044).

\section{Resistance}

Six to nine in vitro passages of A/WSN/33 $(\mathrm{H} 1 \mathrm{~N} 1)$ virus in the presence of baloxavir revealed that an I38T substitution in PA conferred 22- to 41-fold reduced susceptibility to baloxavir (Noshi et al. 2018). The PA I38T substitution occurs infrequently in naturally occurring $\mathrm{A}(\mathrm{H} 1 \mathrm{~N} 1) \mathrm{pdm} 09, \mathrm{~A}(\mathrm{H} 3 \mathrm{~N} 2)$, and $\mathrm{B}$ viruses (0\%-0.02\%) (Stevaert et al. 2013; Takashita et al. 2019b), suggesting that this substitution emerges as a result of treatment.

In a mouse model of highly pathogenic A/Guangdong/17SF003/2016(H7N9) virus infection, emergence of resistance has not been observed (Kiso et al. 2019a). In contrast, baloxavir induced the PA I38M and E199G substitutions associated with reduced susceptibility to baloxavir (Gubareva et al. 2019) in immunocompromised nude mice infected with A/California/04/2009(H1N1)pdm09 virus (Kiso et al. $2019 b$ ), although variants with reduced susceptibility to baloxavir did not emerge in immunosuppressed mice infected with A/Puerto Rico/8/ 34(H1N1) virus (http://www.pmda.go.jp/files/ 000225380.pdf).

In phase 2 and 3 clinical trials of baloxavir, the PA E23K, I38T, and I38F substitutions in A (H1N1)pdm09 viruses and the PA E23G/K,
A37T, I38T, I38M, and E199G substitutions in $\mathrm{A}(\mathrm{H} 3 \mathrm{~N} 2)$ viruses emerged after baloxavir treatment (Hayden et al. 2018; Omoto et al. 2018; Hirotsu et al. 2019). These substitutions conferred 4.5- to 57-fold reduced susceptibility to baloxavir (Omoto et al. 2018). The PA I38 substitution was the most frequently detected mutation and had a greater impact on baloxavir susceptibility than did the other substitutions. Most of the PA I38 substitutions (52.2\%) were detected in children aged $<6 \mathrm{yr}$ infected with $\mathrm{A}(\mathrm{H} 3 \mathrm{~N} 2)$ virus (Table 3). Patients infected with PA I38 mutant viruses exhibited prolonged virus shedding, rebound in virus titers, and delayed symptom alleviation (Hayden et al. 2018; Hirotsu et al. 2019; Uehara et al. 2019).

In Japan, nationwide monitoring of the baloxavir susceptibility of influenza viruses was initiated in the 2017-2018 influenza season (Takashita et al. 2018). During this monitoring, clinical specimens are randomly collected from patients regardless of whether or not they were treated with NA inhibitors or baloxavir (https ://www.niid.go.jp/niid/en/influ-resist-e.html). During the 2018-2019 season, 2.3\% (9/395) of A (H1N1)pdm09 and 8.0\% (34/424) of A(H3N2) viruses were found to possess the PA I38 substitutions (Table 4). Eight of these PA I38 mutant viruses were isolated from patients without prior baloxavir exposure, indicating human-to-human transmission of these mutant viruses (Takashita et al. 2019a,c).

\section{CONCLUDING REMARKS}

Previous studies using laboratory strains demonstrated that A/WSN/33(H1N1) and A/Victoria/3/75(H3N2) viruses with the PA I38T substitution showed decreased susceptibility to baloxavir and reduced replication fitness in vitro (Noshi et al. 2018; Omoto et al. 2018). In contrast, Checkmahomed et al. (2020) reported that the PA I38T substitution did not alter the replication fitness of the contemporary influenza viruses A/Quebec/144147/2009(H1N1)pdm09 and A/Switzerland/9715293/2013(H3N2) in vitro or in mice. Furthermore, A(H1N1)pdm09 and $\mathrm{A}(\mathrm{H} 3 \mathrm{~N} 2)$ viruses with the PA I38T substitution isolated from baloxavir-treated patients 
Influenza Polymerase Inhibitors

Table 3. Influenza viruses with 138 substitutions in PA detected during baloxavir clinical trials

\begin{tabular}{lcccc}
\hline & & \multicolumn{3}{c}{ Type/subtype } \\
\cline { 3 - 4 } Clinical trial & Age group (years) & $\mathrm{A}(\mathrm{H} 1 \mathrm{~N} 1)$ pdm09 & $\mathrm{A}(\mathrm{H} 3 \mathrm{~N} 2)$ & $\mathrm{B}$ \\
\hline $\begin{array}{l}\text { Phase } 3 \text { in Japan } \\
\text { Tablet: T0822 }\end{array}$ & $<6$ & $20.0 \%(1 / 5)$ & $52.2 \%(12 / 23)$ & $0 \%(0 / 13)$ \\
$\quad$ Granule: T0833 & & $0 \%(0 / 2)$ & $18.9 \%(10 / 53)$ & $0 \%(0 / 3)$ \\
$\begin{array}{l}\text { Phase 2: T0821 } \\
\text { Phase 3: T0831 }\end{array}$ & $\geq 12$ & $3.4 \%(4 / 116)$ & $10.3 \%(35 / 341)$ & $0 \%(0 / 87)$ \\
$\quad$ CAPSTONE-1 & & & & \\
(NCT02954354) & & & $7.1 \%(7 / 99)$ & $1.1 \%(1 / 88)$ \\
Phase 3: T0832 & 12 to $<65$ & $7.1 \%(1 / 14)$ & $14.6 \%(6 / 41)$ & $0 \%(0 / 41)$ \\
CAPSTONE-2 & $\geq 65$ & $0 \%(0 / 3)$ & & \\
(NCT02949011) & & & & \\
\hline
\end{tabular}

Data adapted from Shionogi \& Co., Ltd., 1st Half of Fiscal 2019 Financial Results.

Patients with paired baseline and follow-up RT-PCR-positive samples evaluable for Sanger Sequencing (http://www .shionogi.co.jp/en/ir/pdf/e_p191031.pdf).

(PA) Polymerase acidic protein.

during the 2018-2019 influenza season showed similar replication fitness and pathogenicity to those of wild-type isolates in hamsters and transmitted efficiently between ferrets by respiratory droplets (Imai et al. 2020). These results, together with human-to-human transmission cases in Japan, indicate that currently circulating viruses have already acquired amino acid substitutions to compensate for the fitness costs of the PA I38T substitution. Because of the limited data and high frequency of variants with reduced susceptibility to baloxavir, the Japanese Association for Infectious Diseases and the Japan Pediatric Society have not actively recommended use of baloxavir for treatment of influenza in children younger than $12 \mathrm{yr}$ and have not recommended use of baloxavir for monotherapy of influenza in the severely immunosuppressed.
The emergence of antiviral resistance is a great concern. Evidence suggests that the detection of the PA I38T substitution can be considered a laboratory correlate of clinically relevant baloxavir resistance (Gubareva and Fry 2019). Because global surveillance of baloxavir resistance is essential, the World Health Organization (WHO) Global Influenza Surveillance and Response System (GISRS) Expert Working Group for Surveillance of Antiviral Susceptibility (WHO-AVWG) (https://www.who.int/influ enza/gisrs_laboratory/antiviral_susceptibility/ en/) has initiated a global analysis of circulating influenza viruses in the 2017-2018 influenza season (Takashita et al. 2020). The monitoring of baloxavir susceptibility is important for public health planning purposes and for making clinical recommendations for antiviral use.

Table 4. Influenza viruses with I38 substitutions in PA detected in the 2018-2019 influenza season in Japan

\begin{tabular}{lccccccc}
\hline & & \multicolumn{5}{c}{ Age group (years) } \\
\cline { 3 - 7 } & & $0-5$ & $6-11$ & $12-19$ & $20-64$ & $\geq 65$ & Unknown \\
\hline $\begin{array}{l}\text { Type/subtype } \\
\text { A(H1N1) } \\
\text { pdm09 }\end{array}$ & $2.3 \%(9 / 395)$ & $0 \%(0 / 61)$ & $3.4 \%\left(7^{\mathrm{a}} / 203\right)$ & $2.3 \%(1 / 44)$ & $1.6 \%\left(1^{\mathrm{a}} / 63\right)$ & $0 \%(0 / 21)$ & $0 \%(0 / 3)$ \\
$\mathrm{A}(\mathrm{H} 3 \mathrm{~N} 2)$ & $8.0 \%(34 / 424)$ & $\begin{array}{c}8.9 \% \\
\left(10^{\mathrm{a}} / 112\right)\end{array}$ & $12.5 \%\left(16^{\mathrm{a}} / 128\right)$ & $9.6 \%\left(5^{\mathrm{a}} / 52\right)$ & $1.1 \%(1 / 90)$ & $5.6 \%\left(2^{\mathrm{a}} / 36\right)$ & $0 \%(0 / 6)$ \\
$\mathrm{B}$ & $0 \%(0 / 9)$ & $0 \%(0 / 16)$ & $0 \%(0 / 8)$ & $0 \%(0 / 7)$ & $0 \%(0 / 3)$ & $0 \%(0 / 1)$ \\
\hline
\end{tabular}


E. Takashita

\section{ACKNOWLEDGMENT}

This article has been made freely available online courtesy of TAUNS Laboratories.

\section{REFERENCES}

Baranovich T, Wong SS, Armstrong J, Marjuki H, Webby RJ Webster RG, Govorkova EA. 2013. T-705 (favipiravir) induces lethal mutagenesis in influenza A H1N1 viruses in vitro. J Virol 87: 3741-3751. doi:10.1128/JVI.02346-12

Baz M, Carbonneau J, Rheaume C, Cavanagh MH, Boivin G. 2018. Combination therapy with oseltamivir and favipiravir delays mortality but does not prevent oseltamivir resistance in immunodeficient mice infected with pandemic $\mathrm{A}(\mathrm{H} 1 \mathrm{~N} 1)$ influenza virus. Viruses 10: 610. doi:10 3390/v10110610

Byrn RA, Jones SM, Bennett HB, Bral C, Clark MP, Jacobs MD, Kwong AD, Ledeboer MW, Leeman JR, McNeil CF, et al. 2015. Preclinical activity of VX-787, a first-in-class, orally bioavailable inhibitor of the influenza virus polymerase PB2 subunit. Antimicrob Agents Chemother 59: 1569-1582. doi:10.1128/AAC.04623-14

Checkmahomed L, M'Hamdi Z, Carbonneau J, Venable MC, Baz M, Abed Y, Boivin G. 2020. Impact of the baloxavir-resistant polymerase acid I38T substitution on the fitness of contemporary influenza A(H1N1)pdm09 and A(H3N2) strains. J Infect Dis 221: 63-70. doi:10.1093/ infdis/jiz418

Clark MP, Ledeboer MW, Davies I, Byrn RA, Jones SM, Perola E, Tsai A, Jacobs M, Nti-Addae K, Bandarage UK, et al. 2014. Discovery of a novel, first-in-class, orally bioavailable azaindole inhibitor (VX-787) of influenza PB2. J Med Chem 57: 6668-6678. doi:10.1021/jm5007275

Fan H, Walker AP, Carrique L, Keown JR, Serna Martin I, Karia D, Sharps J, Hengrung N, Pardon E, Steyaert J, et al. 2019. Structures of influenza A virus RNA polymerase offer insight into viral genome replication. Nature 573: 287-290. doi:10.1038/s41586-019-1530-7

Finberg RW, Lanno R, Anderson D, Fleischhackl R, van Duijnhoven W, Kauffman RS, Kosoglou T, Vingerhoets J, Leopold L. 2019. Phase 2b study of pimodivir (JNJ63623872) as monotherapy or in combination with oseltamivir for treatment of acute uncomplicated seasonal influenza A: TOPAZ Trial. J Infect Dis 219: 1026-1034. doi:10.1093/infdis/jiy547

Fukao K, Noshi T, Yamamoto A, Kitano M, Ando Y, Noda T, Baba K, Matsumoto K, Higuchi N, Ikeda M, et al. 2019. Combination treatment with the cap-dependent endonuclease inhibitor baloxavir marboxil and a neuraminidase inhibitor in a mouse model of influenza A virus infection. J Antimicrob Chemother 74: 654-662. doi:10.1093/jac/ dky462

Furuta Y, Takahashi K, Fukuda Y, Kuno M, Kamiyama T, Kozaki K, Nomura N, Egawa H, Minami S, Watanabe Y, et al. 2002. In vitro and in vivo activities of anti-influenza virus compound T-705. Antimicrob Agents Chemother 46: 977-981. doi:10.1128/AAC.46.4.977-981.2002

Furuta Y, Takahashi K, Kuno-Maekawa M, Sangawa $H$, Uehara S, Kozaki K, Nomura N, Egawa H, Shiraki K 2005. Mechanism of action of T-705 against influenza virus. Antimicrob Agents Chemother 49: 981-986. doi: 10.1128/AAC.49.3.981-986.2005

Furuta Y, Gowen BB, Takahashi K, Shiraki K, Smee DF, Barnard DL. 2013. Favipiravir (T-705), a novel viral RNA polymerase inhibitor. Antiviral Res 100: 446-454. doi:10.1016/j.antiviral.2013.09.015

Furuta Y, Komeno T, Nakamura T. 2017. Favipiravir (T-705), a broad spectrum inhibitor of viral RNA polymerase. Proc Jpn Acad Ser B Phys Biol Sci 93: 449-463. doi:10.2183/pjab.93.027

Goldhill DH, te Velthuis AJW, Fletcher RA, Langat P, Zambon M, Lackenby A, Barclay WS. 2018. The mechanism of resistance to favipiravir in influenza. Proc Natl Acad Sci 115: 11613-11618. doi:10.1073/pnas.1811345115

Goldhill DH, Langat P, Xie H, Galiano M, Miah S, Kellam P, Zambon M, Lackenby A, Barclay WS. 2019. Determining the mutation bias of favipiravir in influenza virus using next-generation sequencing. J Virol 93: e01217-18. doi:10 .1128/JVI.01217-18

Gubareva LV, Fry AM. 2019. Baloxavir and treatment-emergent resistance: public health insights and next steps. J Infect Dis 221: 337-339.

Gubareva LV, Mishin VP, Patel MC, Chesnokov A, Nguyen HT, De La Cruz J, Spencer S, Campbell AP, Sinner M, Reid H, et al. 2019. Assessing baloxavir susceptibility of influenza viruses circulating in the United States during the 2016/17 and 2017/18 seasons. Euro Surveill 24: 1800666. doi:10.2807/1560-7917.ES.2019.24.3.1800666

Hayden FG, Shindo N. 2019. Influenza virus polymerase inhibitors in clinical development. Curr Opin Infect Dis 32: 176-186. doi:10.1097/QCO.0000000000000532

Hayden FG, Sugaya N, Hirotsu N, Lee N, de Jong MD, Hurt AC, Ishida T, Sekino H, Yamada K, Portsmouth S, et al. 2018. Baloxavir marboxil for uncomplicated influenza in adults and adolescents. New Engl J Med 379: 913-923. doi:10.1056/NEJMoa1716197

Hengrung N, El Omari K, Serna Martin I, Vreede FT, Cusack S, Rambo RP, Vonrhein C, Bricogne G, Stuart DI, Grimes JM, et al. 2015. Crystal structure of the RNA-dependent RNA polymerase from influenza C virus. Nature 527: 114-117. doi:10.1038/nature15525

Hirotsu N, Sakaguchi H, Sato C, Ishibashi T, Baba K, Omoto S, Shishido T, Tsuchiya K, Hayden FG, Uehara T, et al 2019. Baloxavir marboxil in Japanese pediatric patients with influenza: safety and clinical and virologic outcomes. Clin Infect Dis ciz908. doi:10.1093/cid/ciz908

Imai M, Yamashita M, Sakai-Tagawa Y, Iwatsuki-Horimoto K, Kiso M, Murakami J, Yasuhara A, Takada K, Ito M, Nakajima N, et al. 2020. Influenza A variants with reduced susceptibility to baloxavir isolated from Japanese patients are fit and transmit through respiratory droplets. Nat Microbiol 5: 27-33. doi:10.1038/s41564-019-0609-0

Jin Z, Smith LK, Rajwanshi VK, Kim B, Deval J. 2013. The ambiguous base-pairing and high substrate efficiency of T-705 (favipiravir) ribofuranosyl 5' -triphosphate towards influenza A virus polymerase. PLoS One 8: e68347. doi:10 .1371/journal.pone.0068347

Kiso M, Lopes TJS, Yamayoshi S, Ito M, Yamashita M, Nakajima N, Hasegawa H, Neumann G, Kawaoka Y. 2018 Combination therapy with neuraminidase and polymerase inhibitors in nude mice infected with influenza virus. $J$ Infect Dis 217: 887-896. doi:10.1093/infdis/jix606 
Kiso M, Yamayoshi S, Furusawa Y, Imai M, Kawaoka Y 2019a. Treatment of highly pathogenic H7N9 virus-infected mice with baloxavir marboxil. Viruses 11: 1066. doi:10.3390/v11111066

Kiso M, Yamayoshi S, Murakami J, Kawaoka Y. 2019b. Baloxavir marboxil treatment of nude mice infected with influenza A virus. J Infect Dis jiz665. doi: 10.1093/in fdis/jiz665

Koshimichi H, Tsuda Y, Ishibashi T, Wajima T. 2019. Population pharmacokinetic and exposure-response analyses of baloxavir marboxil in adults and adolescents including patients with influenza. J Pharm Sci 108: 1896-1904. doi:10.1016/j.xphs.2018.12.005

Koszalka P, Tilmanis D, Roe M, Vijaykrishna D, Hurt AC. 2019. Baloxavir marboxil susceptibility of influenza viruses from the Asia-Pacific, 2012-2018. Antiviral Res 164: 91-96. doi:10.1016/j.antiviral.2019.02.007

Liu Y, Yang Y, Fan J, He R, Luo M, Zheng X. 2015. The crystal structure of the PB2 cap-binding domain of influenza B virus reveals a novel cap recognition mechanism. J Biol Chem 290: 9141-9149. doi:10.1074/jbc.M115.636464

Marathe BM, Wong SS, Vogel P, Garcia-Alcalde F, Webster RG, Webby RJ, Najera I, Govorkova EA. 2016. Combinations of oseltamivir and T-705 extend the treatment window for highly pathogenic influenza $\mathrm{A}(\mathrm{H} 5 \mathrm{~N} 1)$ virus infection in mice. Sci Rep 6: 26742. doi:10.1038/ srep26742

Mifsud EJ, Hayden FG, Hurt AC. 2019. Antivirals targeting the polymerase complex of influenza viruses. Antiviral Res 169: 104545. doi:10.1016/j.antiviral.2019.104545

Mishin VP, Patel MC, Chesnokov A, De La Cruz J, Nguyen HT, Lollis L, Hodges E, Jang Y, Barnes J, Uyeki T, et al. 2019. Susceptibility of influenza A, B, C, and D viruses to baloxavir. Emerg Infect Dis 25: 1969-1972. doi:10.3201/ eid2510.190607

Noshi T, Kitano M, Taniguchi K, Yamamoto A, Omoto S, Baba K, Hashimoto T, Ishida K, Kushima Y, Hattori K, et al. 2018. In vitro characterization of baloxavir acid, a firstin-class cap-dependent endonuclease inhibitor of the influenza virus polymerase PA subunit. Antiviral Res 160: 109-117. doi:10.1016/j.antiviral.2018.10.008

Omoto S, Speranzini V, Hashimoto T, Noshi T, Yamaguchi H, Kawai M, Kawaguchi K, Uehara T, Shishido T, Naito A, et al. 2018. Characterization of influenza virus variants induced by treatment with the endonuclease inhibitor baloxavir marboxil. Sci Rep 8: 9633. doi:10.1038/ s41598-018-27890-4

Park S, Kim JI, Lee I, Lee S, Hwang MW, Bae JY, Heo J, Kim D, Jang SI, Kim H, et al. 2014. Combination effects of peramivir and favipiravir against oseltamivir-resistant 2009 pandemic influenza $\mathrm{A}(\mathrm{H} 1 \mathrm{~N} 1)$ infection in mice. PLoS One 9: e101325. doi:10.1371/journal.pone.0101325

Peng Q, Liu Y, Peng R, Wang M, Yang W, Song H, Chen Y, Liu S, Han M, Zhang X, et al. 2019. Structural insight into RNA synthesis by influenza D polymerase. Nat Microbiol 4: 1750-1759. doi:10.1038/s41564-019-0487-5

Reich S, Guilligay D, Pflug A, Malet H, Berger I, Crépin T, Hart D, Lunardi T, Nanao M, Ruigrok RW, et al. 2014 Structural insight into cap-snatching and RNA synthesis by influenza polymerase. Nature 516: 361-366. doi:10 $.1038 /$ nature 14009
Sangawa H, Komeno T, Nishikawa H, Yoshida A, Takahashi K, Nomura N, Furuta Y. 2013. Mechanism of action of T-705 ribosyl triphosphate against influenza virus RNA polymerase. Antimicrob Agents Chemother 57: 52025208. doi:10.1128/AAC.00649-13

Severin C, Rocha de Moura T, Liu Y, Li K, Zheng X, Luo M. 2016. The cap-binding site of influenza virus protein PB2 as a drug target. Acta Crystallogr D Struct Biol 72: 245253. doi:10.1107/S2059798316000085

Smee DF, Hurst BL, Wong MH, Bailey KW, Tarbet EB, Morrey JD, Furuta Y. 2010. Effects of the combination of favipiravir (T-705) and oseltamivir on influenza A virus infections in mice. Antimicrob Agents Chemother 54: 126-133. doi:10.1128/AAC.00933-09

Smee DF, Tarbet EB, Furuta Y, Morrey JD, Barnard DL. 2013. Synergistic combinations of favipiravir and oseltamivir against wild-type pandemic and oseltamivir-resistant influenza A virus infections in mice. Future Virol 8: 1085-1094. doi:10.2217/fvl.13.98

Stevaert A, Naesens L. 2016. The influenza virus polymerase complex: an update on its structure, functions, and significance for antiviral drug design. Med Res Rev 36: 11271173. doi:10.1002/med.21401

Stevaert A, Dallocchio R, Dessi A, Pala N, Rogolino D, Sechi M, Naesens L. 2013. Mutational analysis of the binding pockets of the diketo acid inhibitor L-742,001 in the influenza virus PA endonuclease. J Virol 87: 10524-10538. doi:10.1128/JVI.00832-13

Takashita E, Ejima M, Ogawa R, Fujisaki S, Neumann G, Furuta Y, Kawaoka Y, Tashiro M, Odagiri T. 2016. Antiviral susceptibility of influenza viruses isolated from patients pre- and post-administration of favipiravir. Antiviral Res 132: 170-177. doi:10.1016/j.antiviral.2016 .06 .007

Takashita E, Morita H, Ogawa R, Nakamura K, Fujisaki S, Shirakura M, Kuwahara T, Kishida N, Watanabe S, Odagiri T. 2018. Susceptibility of influenza viruses to the novel cap-dependent endonuclease inhibitor baloxavir marboxil. Front Microbiol 9: 3026. doi:10.3389/fmicb.2018 .03026

Takashita E, Ichikawa M, Morita H, Ogawa R, Fujisaki S, Shirakura M, Miura H, Nakamura K, Kishida N, Kuwahara T, et al. 2019a. Human-to-human transmission of influenza $A(\mathrm{H} 3 \mathrm{~N} 2)$ virus with reduced susceptibility to baloxavir, Japan, February 2019. Emerg Infect Dis 25: 2108-2111. doi:10.3201/eid2511.190757

Takashita E, Kawakami C, Morita H, Ogawa R, Fujisaki S, Shirakura M, Miura H, Nakamura K, Kishida N, Kuwahara T, et al. 2019b. Detection of influenza A(H3N2) viruses exhibiting reduced susceptibility to the novel cap-dependent endonuclease inhibitor baloxavir in Japan, December 2018. Euro Surveill 24: 1800698. doi:10 .2807/1560-7917.ES.2019.24.3.1800698

Takashita E, Kawakami C, Ogawa R, Morita H, Fujisaki S, Shirakura M, Miura H, Nakamura K, Kishida N, Kuwahara $\mathrm{T}$, et al. 2019c. Influenza $\mathrm{A}(\mathrm{H} 3 \mathrm{~N} 2)$ virus exhibiting reduced susceptibility to baloxavir due to a polymerase acidic subunit I38T substitution detected from a hospitalised child without prior baloxavir treatment, Japan, January 2019. Euro Surveill 24: 1900170. doi:10.2807/ 1560-7917.ES.2019.24.12.1900170 
E. Takashita

Takashita E, Daniels RS, Fujisaki S, Gregory V, Gubareva LV, Huang W, Hurt AC, Lackenby A, Nguyen HT, Pereyaslov D, et al. 2020. Global update on the susceptibilities of human influenza viruses to neuraminidase inhibitors and the cap-dependent endonuclease inhibitor baloxavir, 2017-2018. Antiviral Res doi:10.1016/j.antiviral.2020 .104718

Tarbet EB, Maekawa M, Furuta Y, Babu YS, Morrey JD, Smee DF. 2012. Combinations of favipiravir and peramivir for the treatment of pandemic influenza A/California/04/ 2009 (H1N1) virus infections in mice. Antiviral Res 94: 103-110. doi:10.1016/j.antiviral.2012.03.001

Tarbet EB, Vollmer AH, Hurst BL, Barnard DL, Furuta Y, Smee DF. 2014. In vitro activity of favipiravir and neuraminidase inhibitor combinations against oseltamivirsensitive and oseltamivir-resistant pandemic influenza A (H1N1) virus. Arch Virol 159: 1279-1291. doi:10 $.1007 / \mathrm{s} 00705-013-1922-1$

Trevejo JM, Asmal M, Vingerhoets J, Polo R, Robertson S, Jiang Y, Kieffer TL, Leopold L. 2018. Pimodivir treatment in adult volunteers experimentally inoculated with live influenza virus: a phase IIa, randomized, double-blind, placebo-controlled study. Antivir Ther 23: 335-344. doi:10.3851/IMP3212
Uehara T, Hayden FG, Kawaguchi K, Omoto S, Hurt AC, De Jong MD, Hirotsu N, Sugaya N, Lee N, Baba K, et al. 2019. Treatment-emergent influenza variant viruses with reduced baloxavir susceptibility: impact on clinical and virologic outcomes in uncomplicated influenza. J Infect Dis 221: 346-355.

Vanderlinden E, Vrancken B, Van Houdt J, Rajwanshi VK, Gillemot S, Andrei G, Lemey P, Naesens L. 2016. Distinct effects of T-705 (favipiravir) and ribavirin on influenza virus replication and viral RNA synthesis. Antimicrob Agents Chemother 60: 6679-6691. doi:10.1128/AAC .01156-16

Wakai C, Iwama M, Mizumoto K, Nagata K. 2011. Recognition of cap structure by influenza $\mathrm{B}$ virus RNA polymerase is less dependent on the methyl residue than recognition by influenza A virus polymerase. J Virol 85: 7504-7512. doi:10.1128/JVI.02375-10

Wang Y, Fan G, Salam A, Horby P, Hayden FG, Chen C, Pan J, Zheng J, Lu B, Guo L, et al. 2019. Comparative effectiveness of combined favipiravir and oseltamivir therapy versus oseltamivir monotherapy in critically ill patients with influenza virus infection. J Infect Dis jiz656. doi:10 $.1093 /$ infdis/jiz656 


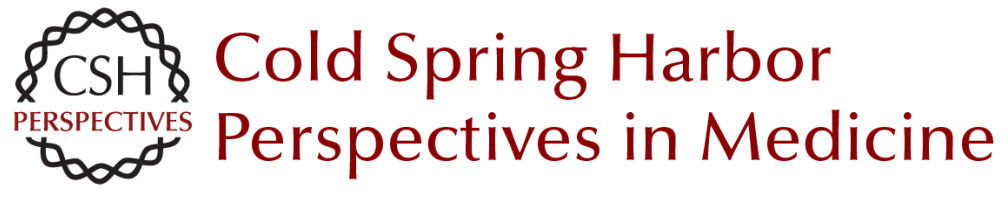

\section{Influenza Polymerase Inhibitors: Mechanisms of Action and Resistance}

Emi Takashita

Cold Spring Harb Perspect Med 2021; doi: 10.1101/cshperspect.a038687 originally published online March 2, 2020

Subject Collection Influenza: The Cutting Edge

Emerging HxNy Influenza A Viruses William J. Liu, Yan Wu, Yuhai Bi, et al.

Equine Influenza

Thomas M. Chambers

Human Influenza Epidemiology

Sukhyun Ryu and Benjamin J. Cowling

Host Cell Factors That Interact with Influenza

Virus Ribonucleoproteins

Ecco Staller and Wendy S. Barclay

Induction and Evasion of Type-I Interferon

Responses during Influenza A Virus Infection

Raquel Muñoz-Moreno, Carles Martínez-Romero and Adolfo García-Sastre

Structure and Function of Influenza Polymerase Joanna M. Wandzik, Tomas Kouba and Stephen Cusack

H7N9 Influenza Virus in China Chengjun Li and Hualan Chen

H5 Influenza Viruses in Egypt Rabeh El-Shesheny, Ahmed Kandeil, Ahmed Mostafa, et al.
Antivirals Targeting the Neuraminidase Larisa Gubareva and Teena Mohan

Accessory Gene Products of Influenza A Virus Rute M. Pinto, Samantha Lycett, Eleanor Gaunt, et al.

Influenza Immunization in the Context of

Preexisting Immunity Susanne L. Linderman, Ali H. Ellebedy, Carl Davis, et al.

Hemagglutinin Structure and Activities Steven J. Gamblin, Sébastien G. Vachieri, Xiaoli Xiong, et al.

Live Attenuated Cold-Adapted Influenza Vaccines Kanta Subbarao

Next-Generation Influenza Vaccines Masaru Kanekiyo and Barney S. Graham

Selective Genome Packaging Mechanisms of Influenza A Viruses

Takeshi Noda

Systems Biological Analysis of Immune Response to Influenza Vaccination

Mario Cortese, Amy C. Sherman, Nadine G.

Rouphael, et al.

For additional articles in this collection, see http://perspectivesinmedicine.cshlp.org/cgi/collection/ 